\title{
ON THE IRREDUCIBILITY OF CERTAIN TRINOMIALS AND QUADRINOMIALS
}

\author{
WILHELM LJUNGGREN
}

1.

The purpose of the present paper is to study the irreducibility over the field of rationals of the polynomials

$$
f(x)=x^{n}+\varepsilon_{1} x^{m}+\varepsilon_{2} x^{p}+\varepsilon_{3},
$$

where $n, m$ and $p$ are natural numbers, $n>m>p$, and $\varepsilon_{1}, \varepsilon_{2} \varepsilon_{3}$, take the values \pm 1 . We can suppose without loss of generality that $n \geqq m+p$, since the roots of $f(x)$ are the inverses of the roots of

$$
x^{n}+\varepsilon_{2} \varepsilon_{3} x^{n-p}+\varepsilon_{1} \varepsilon_{3} x^{n-m}+\varepsilon_{3} .
$$

Further we leave aside the trivial case $n=m+p$ with $\varepsilon_{3}=\varepsilon_{1} \varepsilon_{2}$, where we have the obvious factorization $f(x)=\left(x^{m}+\varepsilon_{2}\right)\left(x^{p}+\varepsilon_{1}\right)$. The complete solution of the problem is given in the following two theorems:

TheOREM 1. If $f(x)$ has no zeros which are roots of unity, then $f(x)$ is irreducible. If $f(x)$ has exactly $q$ such zeros, then $f(x)$ can be decomposed into two rational factors, one of which is of degree $q$ with all these roots of unity as zeros, while the other is irreducible.

Theorem 2. If $n=n_{1} d, \quad m=m_{1} d, \quad p=p_{1} d, \quad$ and $\quad\left(n_{1}, m_{1}, p_{1}\right)=1$ $\left(n_{1}, m_{1}-p_{1}\right)=d_{1}, \quad\left(m_{1}, n_{1}-p_{1}\right)=d_{2}, \quad\left(p_{1}, n_{1}-m_{1}\right)=d_{3}, \quad$ then all possible roots of unity of $f(x)$ are simple zeros, which are to be found among the zeros of

$$
x^{d d_{1}}= \pm 1, \quad x^{d d_{2}}= \pm 1, \quad x^{d d_{3}}= \pm 1 .
$$

In his paper [1] E. S. Selmer has studied the corresponding problem for the trinomials $g(x)=x^{n}+\varepsilon x^{m}+\varepsilon^{\prime}, n \geqq 2 m$, where $\varepsilon$ and $\varepsilon^{\prime}$ take the values \pm 1 , without obtaining the complete solution, apart from the case $m=1$. As a corrolary we find that theorem 1 is valid also if $f(x)$ is replaced by $g(x)$. The corresponding form of theorem 2 was already given by Selmer. See theorem 3 below. We could of course have presented a simpler proof by direct application of the method in this note.

Received April 22, 1960.

Math. Scand. 8 - 5 
2.

Assuming irreducibility of $f(x)$, let

$$
f(x)=\varphi_{r}(x) \psi_{s}(x), \quad r+s=n,
$$

where $\varphi_{r}(x)$ and $\psi_{s}(x)$ are monic polynomials with integral coefficients, of positive degrees $r$ and $s$ respectively.

At first we prove two lemmas.

LEMma 1. In (1) one at least of the two factors must be a reciprocal polynomial.

Proof. Putting

$$
f_{1}(x)=x^{r} \varphi_{r}\left(x^{-1}\right) \psi_{s}(x)=\sum_{i=0}^{n} c_{i} x^{n-i}
$$

we get

$$
f_{2}(x)=x^{s} \psi_{s}\left(x^{-1}\right) \varphi_{r}(x)=x^{n} f_{1}\left(x^{-1}\right)=\sum_{i=0}^{n} c_{n-i} x^{n-i}
$$

and

$$
f_{1}(x) f_{2}(x)=\left(x^{n}+\varepsilon_{1} x^{m}+\varepsilon_{2} x^{p}+\varepsilon_{3}\right)\left(\varepsilon_{3} x^{n}+\varepsilon_{2} x^{n-p}+\varepsilon_{1} x^{n-m}+1\right) .
$$

Equating the coefficients of $x^{2 n}$ and of $x^{n}$ in the two expressions for $f_{1}(x) f_{2}(x)$, we find

$$
c_{0} c_{n}=\varepsilon_{3} \text { and } c_{0}^{2}+c_{1}^{2}+c_{2}^{2}+\ldots+c_{n-1}^{2}=4,
$$

or

$$
c_{0} c_{n}=\varepsilon_{3} \text { and } c_{1}^{2}+c_{2}^{2}+\ldots+c_{n-1}^{2}=2 .
$$

Now (5) implies that two of the $c_{i}$ 's, $i=1,2,3, \ldots, n-1$, say $c_{k_{1}}$ and $c_{k_{2}}$, $k_{1}<k_{2}$, must be equal to \pm 1 , and the other $c_{i}$ 's must be equal to zero.

The first expression for the reciprocal polynomial $f_{1}(x) f_{2}(x)$ is then reduced to

$$
\begin{aligned}
f_{1}(x) f_{2}(x)= & c_{0} c_{n} x^{2 n}+c_{k_{1}} c_{n} x^{2 n-k_{1}}+c_{k_{2}} c_{n} x^{2 n-k_{2}}+ \\
& +c_{0} c_{k_{1}} x^{n+k_{1}}+c_{0} c_{k_{2}} x^{n+k_{2}}+c_{k_{1}} c_{k_{2}} x^{n+k_{2}-k_{1}}+4 x^{n}+\ldots
\end{aligned}
$$

By multiplication (4) yields

$$
\begin{aligned}
f_{1}(x) f_{2}(x)=\varepsilon_{3} x^{2 n}+\varepsilon_{2} x^{2 n-p}+\varepsilon_{1} x^{2 n-m}+ \\
\\
\quad+\varepsilon_{1} \varepsilon_{3} x^{n+m}+\varepsilon_{2} \varepsilon_{3} x^{n+p}+\varepsilon_{1} \varepsilon_{2} x^{n+m-p}+4 x^{n}+\ldots .
\end{aligned}
$$

In order to compare (6) and (7) we shall arrange the exponents of the various powers of $x$, according to their magnitude, in two descending sequences, each containing just the first three terms, For (6) we then get the following four possibilities: 
$(6,1) \quad k_{2} \leqq \frac{n}{2}$

$$
: 2 n>2 n-k_{1}>2 n-k_{2} \text {. }
$$

$(6,2) \quad k_{2}>\frac{n}{2}, \quad k_{1} \leqq n-k_{2} \quad: \quad 2 n>2 n-k_{1} \geqq n+k_{2}$,

$(6,3) \quad k_{2}>\frac{n}{2}, \quad \frac{n}{2} \geqq k_{1}>n-k_{2}: \quad 2 n>n+k_{2}>2 n-k_{2}$,

$(6,4) \quad k_{2}>\frac{n}{2}, \quad k_{1}>\frac{n}{2} \quad: \quad 2 n>n+k_{2}>n+k_{1}$.

For (7) we have only to distinguish between two cases:

$(7,1) \quad n \geqq 2 m$

: $2 n>2 n-p>2 n-m$,

$(7,2) \quad 2 m>n \geqq n+p$

: $\quad 2 n>2 n-p \geqq n+m$.

Combining $(6,1),(6,2),(6,3)$ and $(6,4)$ with $(7,1)$, we find in order the following four possibilities:

$$
\left(k_{1}, k_{2}\right)=(p, m),(p, n-m),(m, n-p) \text { or }(n-m, n-p) .
$$

If $(7,1)$ is replaced by $(7,2)$, the result is

$$
\left(k_{1}, k_{2}\right)=(p, n-m),(p, m),(n-m, n-p) \text { or }(m, n-p) .
$$

Putting $\left(k_{1}, k_{2}\right)=(p, m)$, we obtain from $(6)$ and $(7)$

whence

$$
c_{0} c_{n}=\varepsilon_{3}, \quad c_{p} c_{n}=\varepsilon_{2} \text { and } \quad c_{m} c_{n}=\varepsilon_{1},
$$

$$
f_{1}(x)=c_{n}\left(\varepsilon_{3} x^{n}+\varepsilon_{2} x^{n-p}+\varepsilon_{1} x^{n-m}+1\right)=c_{n} x^{n} f\left(x^{-1}\right),
$$

from which it readily follows that

$$
\psi_{s}(x)=c_{n} x^{s} \psi_{s}\left(x^{-1}\right) .
$$

If $\left(k_{1}, k_{2}\right)=(n-m, n-p)$ we find by the same reasoning

$$
\begin{gathered}
c_{0} c_{n}=\varepsilon_{3}, \quad c_{0} c_{n-m}=\varepsilon_{1} \quad \text { and } \quad c_{0} c_{n-p}=\varepsilon_{2}, \\
f_{1}(x)=c_{0}\left(x^{n}+\varepsilon_{1} x^{m}+\varepsilon_{2} x^{p}+\varepsilon_{3}\right)=c_{0} f(x), \\
\varphi_{r}(x)=c_{0} x^{r} \varphi_{r}\left(x^{-1}\right) .
\end{gathered}
$$

In the cases $\left(k_{1}, k_{2}\right)=(p, n-m)$ and $\left(k_{1}, k_{2}\right)=(m, n-p)$ a closer examination shows that both imply $n=2 m$, the final result being the same as before, i.e. either $\varphi_{r}(x)$ or $\psi_{s}(x)$ is a reciprocal polynomial.

Lemma 2. If $\lambda$ and $\lambda^{-1}$ are both roots of $f(x)$, then we must have one of the following three possibilities: 


$$
\begin{aligned}
& 1^{\circ} \quad \lambda^{n}=-\varepsilon_{3} \quad \text { and } \quad \lambda^{m-p}=-\varepsilon_{1} \varepsilon_{2} \text {, } \\
& 2^{\circ} \quad \lambda^{m}=-\varepsilon_{1} \varepsilon_{3} \text { and } \lambda^{n-p}=-\varepsilon_{2} \text {, } \\
& 3^{\circ} \quad \lambda^{p}=-\varepsilon_{2} \varepsilon_{3} \text { and } \lambda^{n-m}=-\varepsilon_{1} \text {. }
\end{aligned}
$$

Proof. From the two equations

$$
\lambda^{n}+\varepsilon_{1} \lambda^{m}+\varepsilon_{2} \lambda^{p}+\varepsilon_{3}=0, \quad \lambda^{n}+\varepsilon_{2} \varepsilon_{3} \lambda^{n-p}+\varepsilon_{1} \varepsilon_{3} \lambda^{n-m}+\varepsilon_{3}=0
$$

it follows by subtraction

or

$$
\varepsilon_{2} \varepsilon_{3} \lambda^{n-p}+\varepsilon_{1} \varepsilon_{3} \lambda^{n-m}-\varepsilon_{1} \lambda^{m}-\varepsilon_{2} \lambda^{p}=0,
$$

$$
\left(\varepsilon_{2} \lambda^{m-p}+\varepsilon_{1}\right)\left(\varepsilon_{3} \lambda^{n-m}-\varepsilon_{1} \varepsilon_{2} \lambda^{p}\right)=0,
$$

hence either $\lambda^{p}=-\varepsilon_{1} \varepsilon_{2} \lambda^{m}$ or $\lambda^{p}=\varepsilon_{1} \varepsilon_{2} \varepsilon_{3} \lambda^{n-m}$. Inserting these values in the equation $f(\lambda)=0$, we conclude either $\lambda^{n}=-\varepsilon_{3}$ or $\left(\lambda^{m}+\varepsilon_{1} \varepsilon_{2}\right)\left(\lambda^{n-m}+\varepsilon_{1}\right)=0$, from which the lemma readily follows.

3.

In this section we prove our two theorems. If a reciprocal polynomial has a zero $\lambda$, then it has also the zero $\lambda^{-1}$, and theorem 1 now follows immediately from the two lemmas.

Since $(n, m-p)=d d_{1}$, it is possible to find two integers $u$ and $v$ such that $d d_{1}=n u+(m-p) v$, hence $\lambda^{d d_{1}}= \pm 1$. In the same way it is found that $\lambda^{d d_{2}}= \pm 1$ and $\lambda^{d d_{3}}= \pm 1$. Then it remains to show that such a root is always a simple root. Combining the expressions in $1^{\circ}, 2^{\circ}$ and $3^{\circ}$ with the equation

$$
n \lambda^{n}+m \lambda^{m}+p \lambda^{p}=0,
$$

we obtain, omitting some trivial calculations, the following necessary conditions that $\lambda$ should be a multiple root: $n=m+p$ and $\varepsilon_{3}=\varepsilon_{1} \varepsilon_{2}$. But we have left aside this case, and theorem 2 is proved.

4.

Then we shall give the conditions which have to be imposed on $n, m, p, \varepsilon_{1}, \varepsilon_{2}$ and $\varepsilon_{3}$, in order that the two equations in $1^{\circ}$ should be compatible. We restrict ourselves to consider this first case, since the remaining ones give similar conditions.

$$
n_{1} / d_{1} \text { odd, }\left(m_{1}-p_{1}\right) / d_{1} \text { odd and } \varepsilon_{3}=\varepsilon_{1} \varepsilon_{2}: \lambda^{d d_{1}}=-\varepsilon_{3} \text {, }
$$
$n_{1} / d_{1}$ even and $\varepsilon_{3}=-1: \quad \lambda^{d d_{1}}=-\varepsilon_{1} \varepsilon_{2}$, $\left(1^{\circ}, 3\right) \quad\left(n_{1}-p_{1}\right) / d_{1}$ even and $\varepsilon_{1} \varepsilon_{2}=-1: \quad \lambda^{d d_{1}}=-\varepsilon_{3}$. 
5.

Theorem. 3. If $n=n_{1} d, \quad m=m_{1} d, \quad\left(n_{1}, m_{1}\right)=1, \quad n \geqq 2 m$, then the polynomial

$$
g(x)=x^{n}+\varepsilon x^{m}+\varepsilon^{\prime}, \quad \varepsilon= \pm 1, \quad \varepsilon^{\prime}= \pm 1,
$$

is irreducible, apart from the following three cases, where $n_{1}+m_{1} \equiv 0(\bmod 3)$ :

$n_{1}, m_{1}$ both odd, $\varepsilon=1 ; n_{1}$ even, $\varepsilon^{\prime}=1 ; m_{1}$ even, $\varepsilon^{\prime}=\varepsilon, g(x)$ then being a product of the polynomial

$$
x^{2 d}+\varepsilon^{m} \varepsilon^{\prime n} x^{d}+1
$$

and a second irreducible polynomial.

Proof. If $n=2 m$ and $\varepsilon^{\prime}=1$, there is nothing to prove. If $n=2 m$ and $\varepsilon^{\prime}=-1$, or if $n>2 m$, we can make use of our theorems 1 and 2 , noticing that

$$
\left(x^{n}+\varepsilon x^{m}+\varepsilon^{\prime}\right)\left(x^{n}-\varepsilon^{\prime}\right)=x^{2 n}+\varepsilon x^{n+m}-\varepsilon \varepsilon^{\prime} x^{m}-1,
$$

where $2 n>n+m>m$ and in case $n=2 m, \varepsilon_{3} \neq \varepsilon_{1} \varepsilon_{2}$. Here we have

and

$$
\begin{gathered}
(2 n, n+m, m)=(m, n)=d, \\
d_{1}=\left(2 n_{1}, n_{1}\right)=n_{1}, \quad d_{2}=\left(n_{1}+m_{1}, 2 n_{1}-m_{1}\right),
\end{gathered}
$$

$$
d_{3}=\left(m_{1}, n_{1}-m_{1}\right)=1 \text {. }
$$

For $d_{2}$ we find the values 1 or 3 according as $n_{1}+m_{1} \equiv 0(\bmod 3)$ or not. It is obvious that a possible root of unity, $\lambda$ of $g(x)$, cannot satisfy an equation $\lambda^{d}= \pm 1$.

If $d_{2}=1$, then $g(x)$ must be irreducible.

The equations corresponding to case $2^{\circ}$, lemma 2 , are here

or

$$
\lambda^{n+m}=\varepsilon \quad \text { and } \quad \lambda^{2 n-m}=\varepsilon \varepsilon^{\prime}
$$

$$
\lambda^{3 n}=\varepsilon^{\prime} \quad \text { and } \lambda^{3 m}=\varepsilon \varepsilon^{\prime} .
$$

If $d_{2}=3$, we conclude $\varepsilon=1, \lambda^{3 d}=\varepsilon^{\prime}$ or $\varepsilon^{\prime}=1, \lambda^{3 d}=\varepsilon$ or $\varepsilon=\varepsilon^{\prime}, \lambda^{3 d}=\varepsilon^{\prime}$, from which we get the last statement in the theorem.

6.

It is a tedious but straight-forward job to find all cases where $f(x)$ is irreducible. We therefore restrict ourselves to state the following simple result:

If $n_{1}, m_{1}$ and $p_{1}$ are all odd integers, then the polynomials $x^{n}+x^{m}+x^{p} \pm \varepsilon_{3}$ are irreducible.

By means of the simple method used in this paper we may also derive other criteria of irreducibility. However, we don't enter into this here. 


\section{REFERENCE}

1. E. S. Selmer, On the irreducibility of certain trinomials, Math. Scand. 4 (1956), 287-302.

UNIVERSITY OF OSLO, NORWAY 